\title{
Electrochemical Insertion/Extraction of Lithium into/from Vanadium Pentoxide Xerogel in a Propylene Carbonate Solution
}

\author{
Takashi MIURA*, Ken-ichi AOKI and Tomiya KISHI \\ Received June 15, 1990 ; Accepted October 26, 1990
}

\begin{abstract}
The reversibility of an electrochemical insertion/extraction reaction of lithium into/from vanadium pentoxide xerogel $\left(\mathrm{V}_{2} \mathrm{O}_{5} \cdot 1.9 \mathrm{H}_{2} \mathrm{O}\right)$ was investigated in a $1 \mathrm{~mol} \mathrm{dm}^{-3} \mathrm{LiClO}_{4}$-propylene carbonate (PC) solution. Galvanostatic discharge/charge cycling potentials and determination of lithium content in this material showed that $x(=\mathrm{Li} / \mathrm{N}$, atomic ratio $)=c a .0 .16$ of lithium is unrechargeable. The coulombic efficiency for a discharge/charge cycle between $0.16<x<0.36$ was, however, expected to approach to $100 \%$.
\end{abstract}

\section{INTRODUCTION}

As an application of vanadium pentoxide xerogel (VXG, $\mathrm{V}_{2} \mathrm{O}_{5} \cdot n \mathrm{H}_{2} \mathrm{O}$ ), one of transition metal oxide hydrates, to a lithium cell, we have reported previously electrochemical ${ }^{1,2)}$ and chemical ${ }^{3,4)}$ insertion of lithium into this positive pole material. Although electrochemical lithiation of VXG was investigated also by Araki $e t$ al. ${ }^{5}$ ) in a propylene carbonate solution, it still remains as a question whether this reaction is reversible or not.

In this work, the possibility of electrochemical extraction of inserted lithium has been investigated by discharge/charge cycling tests accompanied with quantitative analysis of lithium in VXG.

\subsection{Sample electrodes}

\section{EXPERIMENTAL}

A thin film (thickness $c a .5 \mu \mathrm{m}$, area $c a .1 .5 \mathrm{~cm}^{2}$ ) of VXG having an $n$-value of 1.9 was prepared as in a previous work ${ }^{2)}$. The amount of VXG $(1-2 \mathrm{mg})$ was determined on a digital $\left(10^{-5} \mathrm{~g}\right.$ digit $)$ balance from the weight gain of a nickel substrate after forming a VXG film.

X-ray diffraction from a VXG film was measured in the reflection geometry using $\mathrm{CuK}_{\alpha}$ radiation at 3 deg $<2 \theta<45 \mathrm{deg}(2.95 \mathrm{~nm}>$ lattice spacing $>0.201$ $\mathrm{nm}$ ) with a scanning rate of $0.25 \mathrm{deg}(2 \theta) \mathrm{min}^{-1}$.

\subsection{Electrochemical measurements}

Electrochemical measurements were carried out in a $1 \mathrm{~mol} \mathrm{dm}^{-3} \mathrm{LiClO}_{4}-\mathrm{PC}$ solution at $25^{\circ} \mathrm{C}$, where the potential was referred to a reversible lithium electrode in the same electrolyte ${ }^{2)}$.

An apparent extent of lithiation, $x_{Q}$, was calculated as the ratio of (electron/ $\mathrm{V}$ atom) from the amount of passed charge and the initial weight of a VXG film. A lithiated sample was washed with pure acetonitrile and then dried in a silica gel desiccator at room temperature before analysis.

Department of Applied Chemistry, Faculty of Science and Technology, Keio University (Hiyoshi 3-14-1, Kouhoku-ku, Yokohama, 223 Japan)

Key Words : Vanadium Pentoxide Xerogel, Lithium Secondary Cell

\subsection{Analysis of lithium in VXG}

The known amount of electrochemically lithiated VXG was dissolved in a $0.5 \mathrm{~mol} \mathrm{dm}^{-3} \mathrm{H}_{2} \mathrm{SO}_{4}$ aqueous solution after stripped from the substrate. Flame spectrophotometric analysis of lithium was carried out with a Hitachi 180-50 equipment, where several standard solutions were prepared by dissolving lithium metavanadate $\left(\mathrm{LiVO}_{3}\right)$ in $0.5 \mathrm{~mol} \mathrm{dm}^{-3}$ $\mathrm{H}_{2} \mathrm{SO}_{4}$. A calibration curve based on the emission intensity at $670.8 \mathrm{~nm}$ showed a good linearity and the error in lithium determination was expected to be less than $5 \%$.

\section{RESULTS AND DISCUSSION}

\subsection{Electrochemical insertion of lithium}

A typical galvanostatic discharging curve of a virgin electrode is shown in Fig. 1, where a potential plateau lies at $3.0-2.9 \mathrm{~V}$. As mentioned previouly ${ }^{2)}$, an abrupt drop of a discharging potential at around $x_{\mathrm{Q}}=0.36$ corresponds to the limit of electrochemical lithiation in a PC solution.

Discontinuous jumps of a discharging potential as seen Fig. 1 were observed at around $x_{\mathrm{Q}}=0.12$ and 0.16 for many samples and at $x_{\mathrm{Q}}=0.05$ in some cases. (See also discharging curves in the following Fig. 2.) They do not arise from any experimental mistake but are related probably to the disappearance of a concentration overvoltage in the solid phase as discussed below. In general, however, we might have to take into account other factors such as the electronic property of VXG to discuss these discontinuous potential jumps.

As seen in Fig. 2, the analyzed lithium content, $x_{\text {anal }}(=(\mathrm{Li} / \mathrm{V})$ atomic ratio), of a discharged VXG film ensures that an electrochemical lithiation reaction of VXG

$$
\begin{aligned}
\mathrm{V}_{2} \mathrm{O}_{5} \cdot n \mathrm{H}_{2} \mathrm{O}+2 x \mathrm{Li}^{+}+2 x \mathrm{e}^{-} \\
=\mathrm{Li}_{2 x} \mathrm{~V}_{2} \mathrm{O}_{5} \cdot n \mathrm{H}_{2} \mathrm{O}
\end{aligned}
$$

proceeds with the Faradaic efficiency of $c a .100 \%$ up to at least $x_{\mathrm{Q}}=0.36$. The difference between $x_{\text {anal }}$ and $x_{Q}$ is within $\pm 20 \%$, which may arise mainly from the error in VXG weight either of a virgin film or of a dissolved portion for analysis. 


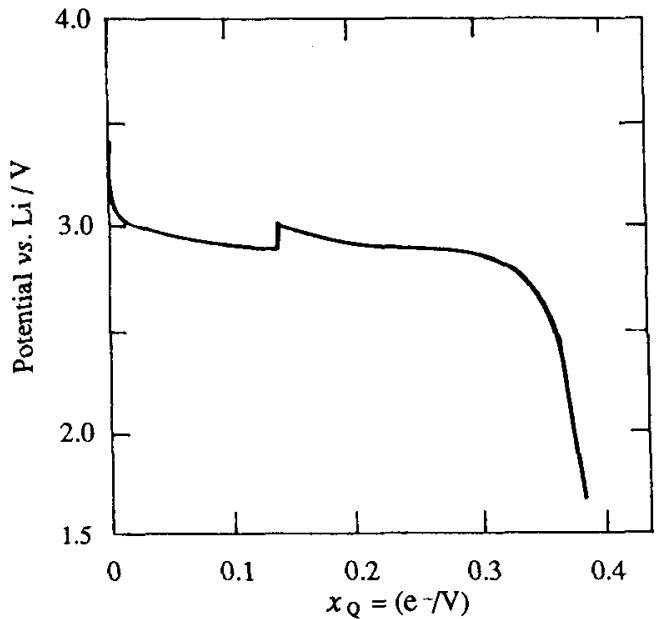

Fig. 1 Typical galvanostatic discharging curve at $-5.4 \mu \mathrm{A}$ $\mathrm{cm}^{-2}$

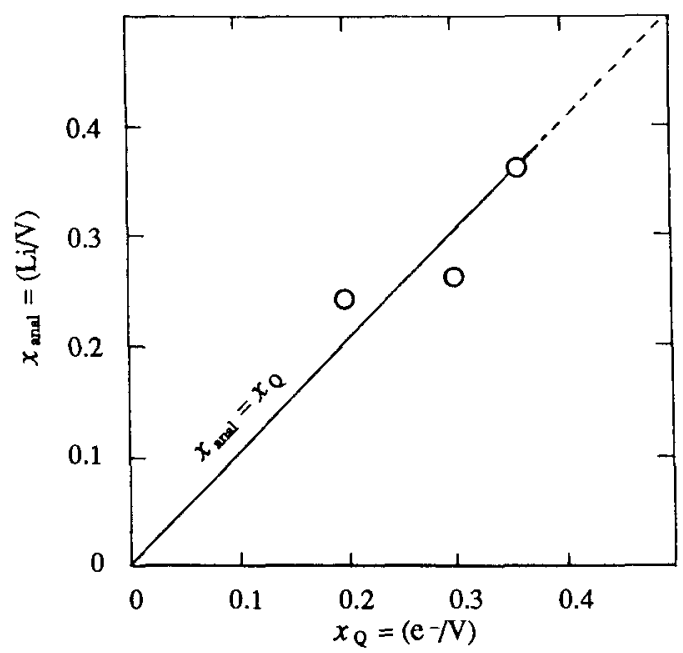

Fig. 2 Relationship between the amounts of discharge electricity and inserted lithium

(galvanostatic discharge at $-5.4 \mu \mathrm{A} \mathrm{cm}^{-2}$ )

3.2 Electrochemical extraction of inserted lithium

Figure 3 shows the first discharge/charge cycling curves reversed at various points of $x_{Q}$ and terminated always at $x_{\mathrm{Q}}=0$. A charging current of +1.6 $\mu \mathrm{A} \mathrm{cm}^{-2}$ was imposed without any pause after discharging. As seen in Fig. 3(D) $-(F)$, the discharging and charging potentials are close to each other in a range of $c a .0 .16<x_{\mathrm{Q}}$ under present experimental conditions. At $x_{\mathrm{Q}}<c a .0 .16$, on the other hand, the charging potential is considerably higher than discharging one (Fig. 3(B) and (C)).

These potential data given in Fig. $3(\mathrm{~B})$ - (F) suggest that cathodically inserted lithium is extractable anodically in the region of ca. $0.16<x_{\mathrm{Q}}$. This implies also that a new phase distinct from VXG host is formed on discharging at around $x_{\mathrm{Q}}=0.16$, in
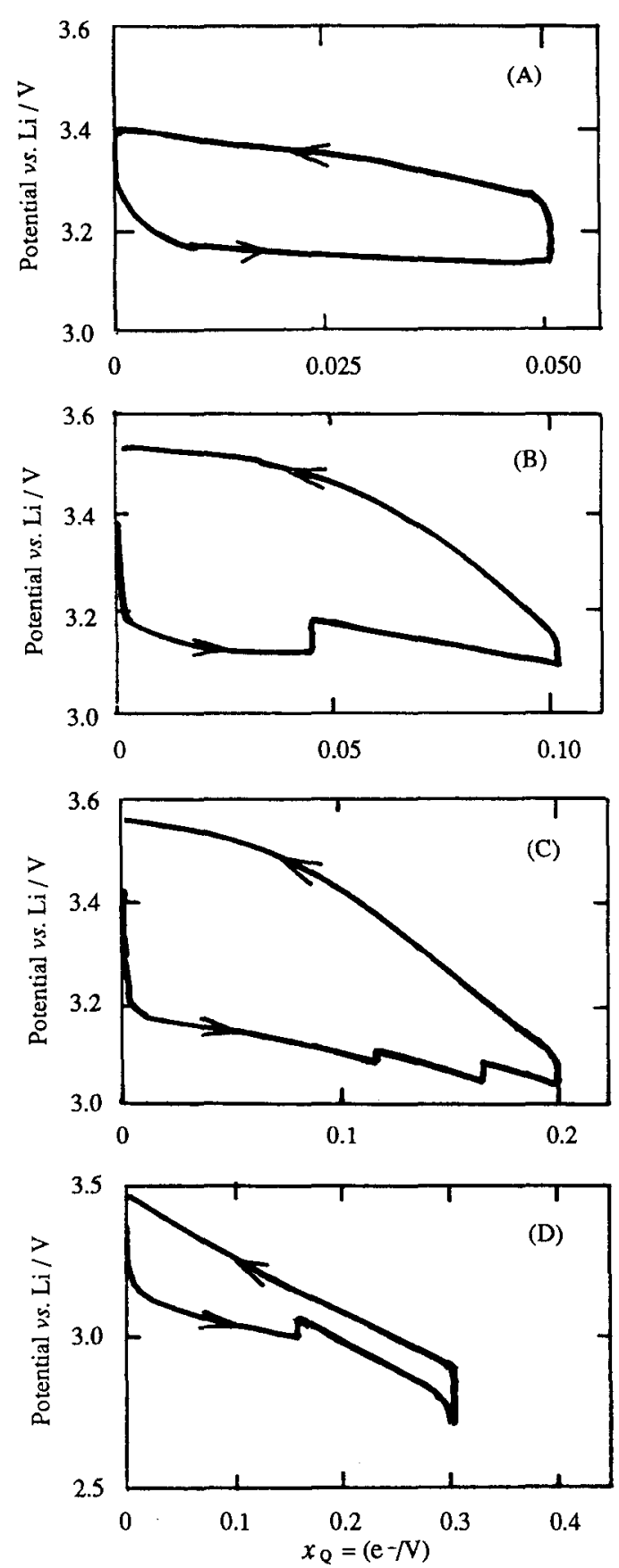

[Figure 3 continues to the next page.]

which both insertion and extraction of lithium can proceed quantitatively. The occurrence of a new phase at $x_{Q}=c a .0 .16$ is related to the discontinuous jumps of a discharging potential as mentioned above, where the concentration overvoltage in the solid phase disappears with the nucleation of a lithiumcontaining new phase. 

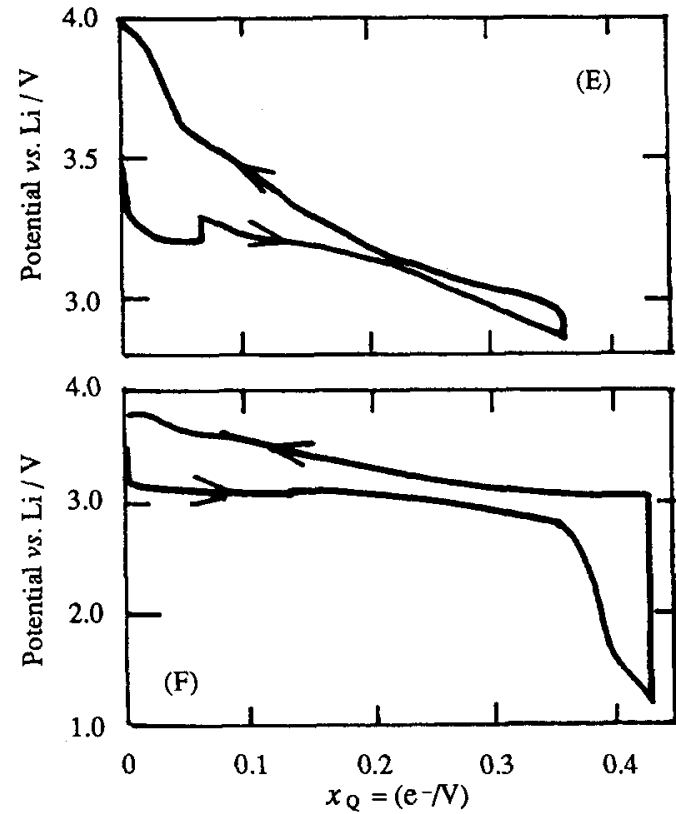

Fig. 3 Galvanostatic discharge $\left(-5.4 \mu \mathrm{A} \mathrm{cm}^{-2}\right) /$ charge $\left(+1.6 \mu \mathrm{A} \mathrm{cm}^{-2}\right)$ cycling curves of a virgin electrode

\begin{tabular}{|lcl|}
\hline & $x_{Q}$ reversed & $x_{\text {anal }}$ \\
\hline (A) & 0.005 & 0.021 \\
$(B)$ & 0.10 & 0.042 \\
$(C)$ & 0.20 & 0.14 \\
(D) & 0.30 & 0.23 \\
(E) & 0.36 & 0.16 \\
(F) & 0.43 & 0.20 \\
\hline
\end{tabular}

As can be seen in Fig. 3(A), insertion/extraction of lithium seems to proceed almost reversibly within a narrow lithiation range of $0 \leq x_{Q} \leq 0.05$. Hence, VXG might be expected to behave as a solid solution electrode in this range of stoichiometry, which is not the case from a structural point of view as mentioned later in 3.4.

The residual amount of lithium after one discharge/charge cycle is given in a caption of Fig. 3 . These analytical data prove clearly that there remains (at least electrochemically) unextractable lithium in VXG. After deep discharge/charge cyclings reversed at $0.16<x_{Q}$ (Fig. 3(C) $-(\mathrm{F})$ ), the residual amount of lithium can be taken as $x_{\text {anal }}=0.16$ in average, though it has a considerable dispersion. Why lithium is unextractable at $x_{\mathrm{Q}}<0.16$ is not clear at present. We suspect, however, that a kinetic factor rather than structural one is responsible to the residence of lithium in this charging region, since charging potentials at $x_{\mathrm{Q}}<0.16$ depend substantially on a current density. From these results, we may conclude that reversible insertion/extraction of lithium into/from VXG is possible in a range of $0.16<x_{Q}<0.36$.

\subsection{Cyclic voltammograms}

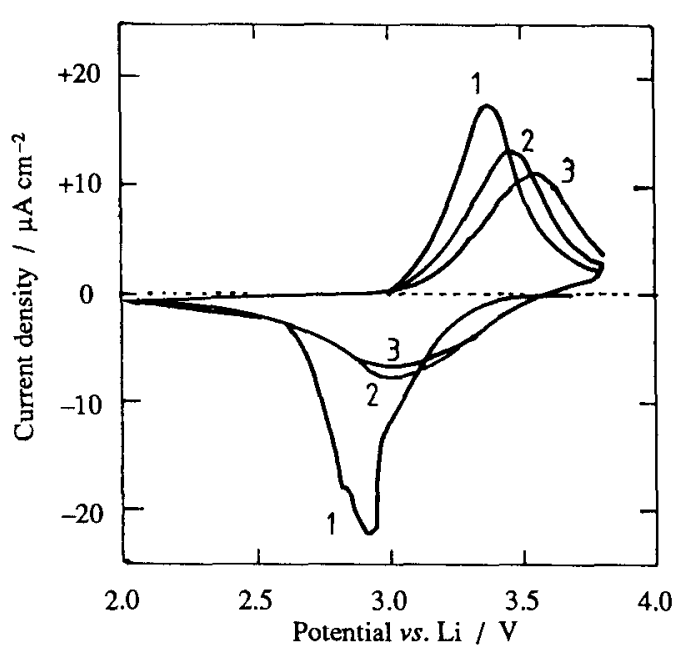

Fig. 4 Typical cyclic voltammogram with a scanning rate of $\pm 2.1 \mu \mathrm{V} \mathrm{s}^{-1}$ (Weight of a VXG film : $1.52 \mathrm{mg}$ )

The presence of unrechargeable lithium in VXG is supported also by coulombic data on cyclic voltammograms started cathodically from an open-circuit potential with an extremely low sweep rate. In Fig. 4, the cathodic charge amounts to $x_{\mathrm{Q}}=0.38,0.19$ and 0.18 for the 1st, 2nd and 3rd sweeps, respectively, whereas the anodic charge amounts to $x_{\mathrm{Q}}=0.21$, 0.19 and 0.16 for the $1 \mathrm{st}$, 2nd and 3rd sweeps, respectively. That is, $x=c a .0 .16$ of lithium remains in VXG at the end of the 1st anodic recharging sweep. Then, in the succeeding 2 nd and 3 rd potential sweep cycles, $\Delta x=c a .0 .20$ of lithium is expected to be inserted/ extracted reversibly between a stoichiometric range of $0.16<x<0.36$.

The cathodic and anodic current peaks lie at around 3.0 and $3.5 \mathrm{~V}$, respectively, both in the 2nd and 3rd cycles.

\subsection{Changes in interlayer gap}

The structural changes of VXG accompanied with electrochemical lithiation in a PC electrolyte ${ }^{2)}$ or with chemical lithiation in acetonitrile solutions ${ }^{4)}$ were reported previously in detail. In this paper, XRD results of a VXG film after complete galvanostatic cycling as in Fig. 3 will be discussed briefly.

The appearance of new phases as expected from discontinuous discharging curves can not be confirmed on a diffractogram even after deep discharging at $0.4<x_{Q}$. Instead, all observed diffraction peaks from a VXG film both in discharged and completely recharged (on a coulombic basis) states can be assigned as $(00 l)$ harmonics, although their intensities are extremely low $(c a .1 / 100)$ compared with those from a virgin film.

The interlayer gap, $d_{001}$, calculated as an average value from each diffraction angle of $(00 l)(l=1,3,4,5)$ harmonics is plotted in Fig. 5 against $x_{Q}$, which corresponds to the end point of discharging or the 


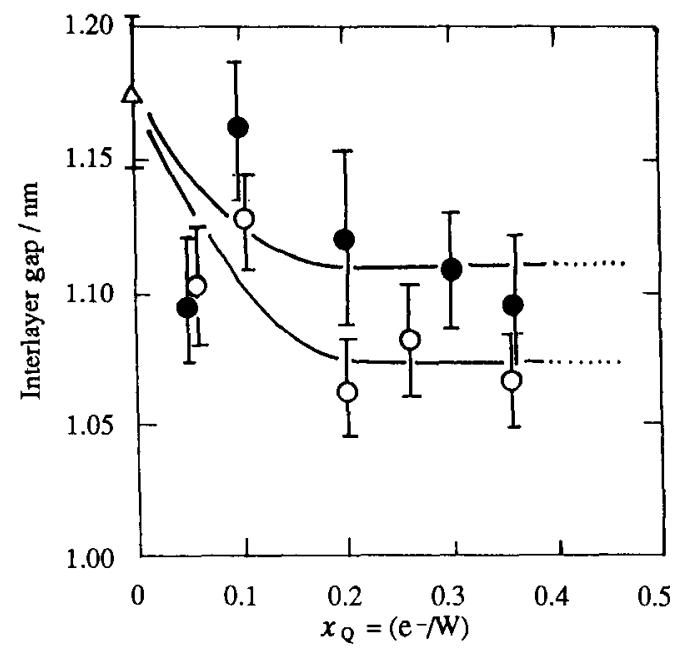

Fig. 5 Changes in the interlayer gap caused by discharging/charging (See text)

0 : after discharging

- : after discharge/charge cycling as in Fig. 3

reversed point of complete discharge/charge cycling. The interlayer gap once decreased on discharging does not recuperate completely to the original spacing $(1.17 \mathrm{~nm})$ of a virgin VXG film, which should be related closely to the presence of unextractable lithium.

After deep cycling reversed at $0.16<x_{\mathrm{Q}}, d_{001}$ is roughly constant at $1.10 \mathrm{~nm}$, where the partial recovery of the interlayer gap on recharging may be recognized.

These facts suggest that a lamellar structure of VXG is kept principally during both discharging and discharge/charge cycling, although the structural order along $<001>$ direction perpendicular to the substrate surface is lowered extremely. Thus, the occurrence of a new phase at $x=0.16$ (and probably at $x=0.05$ and 0.12 ) leads to no destruction of the layered structure itself but presumably to the formation of a certain structural unit within an interlayer gap. In-situ XRD study may be desirable for further investigation, since interlayer molecules such as
$\mathrm{H}_{2} \mathrm{O}$ and $\mathrm{PC}$ are possible to escape to circumstances before instrumental analysis.

\section{CONCLUSIONS}

At least one new phase may be formed on discharging at around $(\mathrm{Li} / \mathrm{V})=0.16$, in which further electrochemical insertion/extraction of lithium can proceed reversibly in a stoichiometric range of $0.16<(\mathrm{Li} / \mathrm{V})$ $<0.36$. Thus, the possibility of VXG as a positive pole material in a lithium secondary cell may be limited within this narrow range of $(\mathrm{Li} / \mathrm{V})$ ratio. Probably, the fundamental layered structure of VXG is maintained even after the appearance of this phase, although one-dimensional structural order is irreversibly lowered on discharging.

Since the presence of propylene carbonate molecules has been confirmed in electrochemically lithiated $\mathrm{VXG}^{2)}, \mathrm{PC}$ (and also water) molecules located between layers are expected to play an important role through the interaction with inserted $\mathrm{Li}^{+}$ion. In a succeeding papaer, the effects of interlamellar substitution of water with PC on the electrochemical behaviours will be reported.

This work was supported financially by the Ministry of Education Science and Culture under Subject No. 63550612 (Grant-in-Aid for Scientific Research).

\section{REFERENCES}

1) T. Miura, E. Sugiura, T. Kishi and T. Nagai, Denki Kagaku 56, 413 (1988).

2) T. Miura, S. Kunihiro, Y. Muranushi and T. Kishi, Denki Kagaku 57, 393 (1989).

3) T. Miura, N. Kanamori, Y. Muranushi and T. Kishi, Denki Kagaku 57, 29 (1989).

4) T. Miura, N. Kanamori, Y. Muranushi and T. Kishi, Denki Kagaku 57, 33 (1989).

5) B. Araki, C. Mailhe, N. Baffier, J. Livage and J. Vedel, Solid State Ionics 9-10, 439 (1983). 